\title{
Campanhas de promoção de saúde ocular: experiência do Hospital Banco de Olhos de Porto Alegre
}

\author{
Ocular health promotion: the Hospital Banco \\ de Olhos de Porto Alegre experience
}

Aline Lütz de Araújo', Nicholas Miranda Zucchetto', João Borges Fortes Filho²

\section{Resumo}

Objetivos: Campanhas de promoção de saúde ocular têm sido promovidas por inúmeras instituições de ensino médico numa freqüência crescente devido à grande demanda de pacientes e necessidade de promoção e prevenção da saúde visual. Estas campanhas devem ser formuladas e planejadas previamente, com alocação de pessoal, recursos e logística de atendimento. Este estudo objetiva analisar três campanhas de promoção de saúde ocular, identificando os fatores necessários para que tais eventos atinjam seus objetivos sociais. Métodos: Estudo observacional descritivo, incluindo três campanhas de atendimento oftalmológico realizadas no ano de 2005. Resultados: As campanhas incluídas foram: (1) Atendimento oftalmológico aos alunos e familiares de uma escola pública, com triagem feita na escola e encaminhamento de 90 pacientes ao hospital. Observou-se alto índice de absenteísmo $(45,5 \%)$ à consulta hospitalar. Foram prescritos óculos a $73 \%$ dos pacientes e $14,5 \%$ necessitaram de encaminhamento para exames ou reconsultas; (2) Screening e informação sobre glaucoma, realizado em uma feira de produtos agrícolas. Foram examinados 107 indivíduos e não foi detectado nenhum caso suspeito; (3) Avaliação da acuidade visual e refração de escolares carentes, realizado no hospital, sem triagem prévia, em 1.200 crianças. Foram prescritos e doados óculos a 18,83\% dos atendidos. Conclusão: Para a realização destas campanhas devem ser consideradas algumas variáveis como: local de atendimento (comunidade ou hospital) e disponibilidade de transporte, que influenciam o comparecimento da população; triagem prévia; disponibilidade de equipamentos para o atendimento necessário; possibilidade de referência dos pacientes para serviço especializado, entre outros. A análise destes fatores revelou diferença na resolutividade das mesmas.

Descritores: Promoção da saúde; Triagem; Saúde ocular;Acuidade visual; Saúde escolar

\footnotetext{
${ }_{2}^{1}$ Residentes do curso de Especialização em Oftalmologia do Hospital Banco de Olhos de Porto Alegre (RS) - Brasil;

2Professor de Oftalmologia da Faculdade de Medicina da Universidade Federal do Rio Grande do Sul - UFRGS; Coordenador do Curso de Especialização em Oftalmologia do Hospital Banco de Olhos de Porto Alegre (RS) - Brasil; Pós-graduando em Oftalmologia pela Escola Paulista de Medicina da Universidade Federal de São Paulo - UNIFESP - São Paulo (SP) - Brasil.
} 


\section{INTRODUÇÃO}

C ampanhas de prevenção da cegueira ou de promoção de saúde ocular têm sido realizadas por muitas instituições de ensino da Oftalmologia em freqüência crescente nos últimos anos. Estes projetos proporcionam a integração entre os alunos, médicos residentes em oftalmologia, diretamente com uma população mais carente e, muitas vezes, à parte do atendimento usual oferecido pelo Sistema Único de Saúde. Para oferecer um bom atendimento durante as campanhas é preciso um planejamento articulado que compreende variáveis como triagem, transporte de pacientes, logística de atendimento, distribuição de material informativo, convênios com laboratórios ou instituição beneficentes para doação de medicações ou óculos necessários, e possibilidade de encaminhamento de pacientes a centros especializados.

Este trabalho tem como objetivos analisar as campanhas de atendimento oftalmológico realizadas pelos alunos do Curso de Especialização em Oftalmologia do Hospital Banco de Olhos de Porto Alegre durante o ano de 2005, identificando os fatores necessários para uma campanha satisfatória e verificando as falhas no seu andamento, bem como traçar o perfil dos pacientes atendidos nestes eventos.

\section{Métodos}

Estudo observacional descritivo baseado em um levantamento de dados das três campanhas de promoção de saúde ocular organizadas no ano de 2005. Os dados para este estudo foram obtidos por meio de fichas de atendimento previamente elaboradas que foram preenchidas pelos médicos durante os atendimentos. Foram excluídos do estudo os indivíduos que não tiveram suas fichas de atendimento adequadamente preenchidas.

As campanhas consideradas para este estudo foram:

(1) Campanha de atendimento oftalmológico destinada aos alunos e familiares de uma escola da rede pública. Esta campanha teve triagem prévia de pacientes baseada na medida da acuidade visual (AV) com tabela de Snellen.A triagem foi feita na própria escola por duas auxiliares de enfermagem da instituição sede do curso de especialização e que foram previamente treinadas para esta finalidade. Os alunos ou familiares que apresentaram AV igual ou inferior a 0,9 na tabela de Snellen durante a triagem foram encaminhados para exame oftalmológico completo realizado no ambulatório do curso de especialização, distante $22 \mathrm{~km}$ do local onde se situava a escola.

(2) Campanha para divulgação da importância de se detectar precocemente o glaucoma, realizada em uma feira de produtos agrícolas na cidade de Porto Alegre, incluiu avaliação do conhecimento da população sobre a doença e orientação quanto a medidas preventi- vas. $\mathrm{O}$ atendimento foi realizado em um consultório montado na própria feira. Os pacientes foram examinados pelos alunos do curso de especialização com medição da AV por tabela de Snellen, exame de tonometria de aplanação com tonômetro de Perkins e exame de fundo de olho por oftalmoscopia monocular direta após dilatação pupilar com uma gota de colírio de tropicamida a $1 \%$. Foram considerados como suspeitos de possuir glaucoma todos os pacientes com pressão intra-ocular igual ou maior do que $21 \mathrm{mmHg}$ pela tonometria de aplanação ou os que apresentaram escavações papilares de 0,5 ou maior em pelo menos um dos olhos ou assimetria das escavações papilares maior do que 0,2.

(3) Avaliação da AV e prescrição de óculos para escolares carentes em parceria com a Prefeitura Municipal de Porto Alegre com atendimento de crianças até a idade de 10 anos sem triagem prévia. Nesta campanha, todos os atendimentos foram realizados no ambulatório do curso de especialização. Não houve triagem prévia. Foram atendidos todos os pacientes que solicitaram atendimento à Secretaria Municipal de Educação em Porto Alegre. Os pacientes, sem triagem prévia, foram transportados até o ambulatório do hospital por meio de ônibus disponibilizado pela própria escola. No ambulatório do curso de especialização todos os pacientes realizaram exame oftalmológico completo.

O banco de dados e a análise estatística dos resultados, baseados em percentuais simples, médias e desvios padrões, foram realizados através do programa MS Excel 2000.

\section{Resultados}

(1) Campanha de atendimento oftalmológico destinada aos alunos e familiares de uma escola da rede pública. De um número não disponível de pacientes previamente triados foram selecionados 90 pacientes para realizarem exame oftalmológico completo na instituição, localizada a $22 \mathrm{~km}$ do local onde a grande maioria dos pacientes vivia (cidade de Esteio no RS). Apenas 49 pacientes (54,4\% dos selecionados na triagem) compareceram ao atendimento no hospital, sendo que apenas 6 pacientes eram estudantes com média de idade de 14,2 anos $( \pm 2,9)$. Os demais 43 pacientes selecionados e que compareceram ao exame eram familiares dos alunos e tinham média de idade de 47,9 anos $( \pm 12,7)$ sendo a maioria $(83,3 \%)$ do sexo feminino.

No exame oftalmológico, $85 \%$ apresentavam AV inferior a 0,9 em pelo menos um dos olhos. Foram prescritos óculos a $73 \%$ dos pacientes e $14,5 \%$ necessitaram encaminhamento para consulta oftalmológica eletiva por necessidade de exames e/ou de tratamento clínico ou cirúrgico.

(2) Campanha para divulgação da importância de se detectar precocemente o glaucoma. Esta prestação de serviço à comunidade foi realizada em uma feira 
Tabela 1

Resumo das Campanhas de Promoção de Saúde Ocular realizadas pelos alunos do Curso de Especialização em Oftalmologia no ano de 2005

\begin{tabular}{ccccccc}
\hline Campanha & $\begin{array}{c}\text { Número de } \\
\text { atendimentos } \\
(\%)\end{array}$ & $\begin{array}{c}\text { Idade } \\
\text { (Média/DP) }\end{array}$ & $\begin{array}{c}\text { Triagem } \\
\text { prévia }\end{array}$ & $\begin{array}{c}\text { Local do } \\
\text { atendimento }\end{array}$ & $\begin{array}{c}\text { Comparecimento } \\
\text { ao atendimento }\end{array}$ & Resolutividade \\
\hline 1 & $49(54,4 \%)$ & $47,9 \operatorname{anos}( \pm 12,7)$ & Sim & Ambulatorial & $54,4 \%$ & $\begin{array}{c}73 \% \text { receberam } \\
\text { prescrição de óculos; } \\
14,5 \% \text { referidos } \\
\text { para nova consulta }\end{array}$ \\
2 & 107 & 37,4 anos $( \pm 15,3)$ & Não & No evento & $100 \%$ & $\begin{array}{c}\text { Nenhum caso } \\
\text { de glaucoma suspeito } \\
18,83 \% \text { receberam } \\
\text { prescrição de óculos; } \\
7,16 \% \text { referidos para } \\
\text { nova consulta }\end{array}$ \\
\hline
\end{tabular}

Campanha 1: Triagem prévia selecionou os casos que necessitavam de atendimento; Atendimento no hospital gerou muitas faltas às consultas;

Campanha 2: Atendimento no evento com poucos recursos de equipamentos;

Campanha 3: Ausência de triagem prévia gerou muitos atendimentos desnecessários; Transporte direto da escola ao hospital favoreceu o índice de comparecimento.

de produtos agrícolas na cidade de Porto Alegre e incluiu avaliação do conhecimento da população sobre a doença e orientação quanto a medidas preventivas. Foram atendidos 107 indivíduos, sendo 80,3\% do sexo masculino e com média de idade de 37,4 anos $( \pm 15,3)$. Não foram detectados casos suspeitos de glaucoma.

(3) Avaliação da AV e prescrição de óculos para escolares carentes em parceria com a Prefeitura Municipal de Porto Alegre. Esta campanha atendeu 1.200 crianças com média de idade de 7 anos $( \pm 2,9)$ que não haviam sido previamente triadas. Foram prescritos e doados óculos a 18,83\% dos pacientes, após exame oftalmológico completo, realizado no ambulatório do curso, em ambiente hospitalar. Constatou-se a necessidade de encaminhar 7,16\% das crianças para nova avaliação ambulatorial.

A tabela 1 ilustra características e observações sobre os resultados obtidos nas três campanhas estudadas.

\section{Dıscussão}

Duas das 3 campanhas descritas foram direcionadas para escolares, a fim de se detectar casos de redução da AV nesta população. A deficiência visual nesta idade interfere no processo de aprendizagem e no desenvolvimento psicossocial da criança ${ }^{(1)}$, e daí decorre a importância do exame nas escolas. Ao verificar a associação entre rendimento escolar e AV, um estudo encontrou prevalência de baixa visão em $80,5 \%$ das crianças com dificuldades na escola cujos professores suspeita- ram de problemas oculares ${ }^{(2)}$. O erro de refração não corrigido é a principal causa de AV reduzida entre esco$\operatorname{lares}^{(1,3)}$, sendo por este motivo que as campanhas são focadas principalmente na refração.

A primeira campanha descrita contou com triagem feita na escola por agentes de saúde previamente treinados (dois técnicos de enfermagem do hospital, devidamente capacitados para aferir AV com tabelas de Snellen). A triagem prévia baseada na AV é um fator facilitador para o atendimento em massa, pois seleciona para o exame oftalmológico apenas os pacientes nos quais se detectou a redução da AV, assim um maior número de pessoas é beneficiado. Esta campanha mostrou que $85 \%$ dos pacientes, previamente triados, tinham AV inferior a 0,9 ; foram prescritos óculos a $73 \%$ dos préselecionados e 14,5\% dos pré-selecionados necessitaram de outros exames por diferentes diagnósticos. Por outro lado, a campanha que atendeu 1.200 crianças no hospital não contou com triagem prévia, acarretando maior tempo dispendido no atendimento, além de maior custo com pessoal e transporte. A maioria dos escolares atendidos tinha visão normal e não apresentava outros problemas oculares. Do ponto de vista de Saúde Pública, é necessário considerar a limitação de recursos assistenciais especializados em nosso meio ${ }^{(4)}$, portanto, a realização de triagem com critérios de encaminhamento deve ser prioridade em uma campanha.

A AV é reconhecidamente o indicador ideal da função visual, sendo a sua medida a recomendação da Organização Mundial da Saúde nas diretrizes para 
erradicar causas preveníveis e curáveis de cegueira ${ }^{(5)}$. Um estudo comparando diferentes testes de função visual (AV para perto, para longe, teste de sensibilidade ao contraste e teste macular de Amsler) concluiu que a AV para perto e longe, quando alterada, se correlaciona melhor com a presença de doença ocular, independentemente da presença de erros refrativoss ${ }^{(6)}$.

O método de triagem com o emprego da medida da AV como teste deve ser breve, econômico e simples, de maneira que possa ser efetuado por pessoa não especialista $^{(7)}$. Entretanto, a acurácia da AV medida por professor ou agente de saúde deve ser levada em consideração. Recomenda-se que o ponto de corte para encaminhamento ao cuidado oftalmológico seja de AV igual a 0,7 ou pior, a fim de diminuir os encaminhamentos desnecessários (falso-positivos), mas ao mesmo tempo evitando a não referência de indivíduos com deficiência visual (falsonegativos $)^{(4)}$. As consultas no hospital revelaram $85 \%$ de AV reduzida na primeira campanha, apesar de todos os pacientes terem sido encaminhados como portadores de redução da $\mathrm{AV}$, conforme aferido pela triagem. Assim, observou-se índice de falso-positivos de $15 \%$. Outros estudos constataram taxas de falso-positivos bastante superiores, de $54,5 \%$ a $59,7 \%{ }^{(1,8)}$, prejudicando o atendimento por muitos encaminhamentos desnecessários.

Quando a campanha é realizada distante do local onde vive o paciente, exigindo que o indivíduo se desloque para ser atendido, corre-se o risco de cobrir apenas parte da população selecionada. Isto ocorreu por ocasião da primeira campanha onde o local da triagem distava $22 \mathrm{~km}$ do local do atendimento e apenas 53,3\% dos pacientes previamente triados compareceram ao exame ambulatorial no dia agendado, ainda que todos os selecionados para as consultas apresentassem queixa oftalmológica e AV abaixo de 0,9 conforme aferido pelo pessoal da equipe de triagem.

Um estudo norte-americano, com triagem da população na comunidade e agendamento de consultas oftalmológicas nos casos selecionados, observou que apenas $41 \%$ de 1.331 indivíduos agendados compareceram à consulta. Os autores ressaltam que, mesmo tendo sido oferecido transporte e consulta gratuitas, as justificativas para o não-comparecimento dos pacientes foram: não recebimento da marcação de consulta, esquecimento, falta de transporte e falta de cobertura pelo plano de saúde ${ }^{(9)}$. Outro estudo com pré-escolares mostrou índice de não comparecimento de 20,6\%, apesar de exaustivos apelos aos pais $^{(10)}$. São possíveis causas para o elevado índice de absenteísmo: insuficiente estímulo ao comparecimento pela equipe de triagem, indisponibilidade de transporte gratuito e hospital de referência longe do local onde foi feita a triagem. Na campanha em que foram atendidas 1.200 crianças, as mesmas foram transportadas até o local do atendimento por ônibus privativos de suas escolas e não ocorreu um não-comparecimento, exceto quando al- guns escolares faltaram às aulas naquele dia, o que não foi possível para este estudo determinar.

$\mathrm{O}$ atendimento no hospital possibilita um exame oftalmológico completo, pois as instalações oferecem todos os equipamentos que podem ser necessários no exame. Estes recursos dificilmente poderiam ser levados até as comunidades.

Na campanha realizada na feira agrícola, observou-se menor resolutividade no atendimento que nas demais campanhas realizadas no ambulatório, pois detectou problemas oculares, mas não se pôde resolvê-los, como através da prescrição de óculos, quando necessário. Esta campanha tinha como objetivo principal difundir conhecimento sobre a cegueira causada por glaucoma e buscava a identificação de pacientes com esta morbidade. Foi focada para a detecção objetiva de alterações oculares através da medida da AV, tonometria e fundoscopia realizados no mesmo dia e no próprio local onde se realizava o evento, mas vários pacientes tiveram que ser encaminhados para segundo atendimento para manejo de outras situações encontradas. Não foram encontrados casos suspeitos de glaucoma, apesar da prevalência importante observada em outros programas de detecção de glaucoma: 7,3\% a 7,8\% ${ }^{(11-12)}$. A prevalência de glaucoma aumenta com o avanço da idade. Os participantes desta campanha, 107 indivíduos, tinham média de idade de 37,4 anos e isto pode justificar o fato de que o atendimento não detectou casos suspeitos de glaucoma entre os examinados. Este atendimento foi dirigido a trabalhadores do setor agrícola do Rio Grande do Sul, o que apontou para uma amostra pouco fidedigna da população em geral em risco para o glaucoma. De qualquer modo, este evento teve também uma finalidade educativa que mostrou ótimo resultado, ou seja, difundir o conhecimento sobre o glaucoma e sua prevenção. Todos os pacientes receberam orientação sobre a importância de consultas periódicas, inclusive para crianças, e quanto à procura por atendimento, quando notada qualquer alteração ocular. Esta formação de mentalidade preventiva na população, relativa às causas de cegueira e à maneira de evitá-las, deve ser prioritária, uma vez que a saúde decorre também de decisão pessoal, e não do simples fato de se ter acesso aos serviços de saúde ${ }^{(5)}$.

Recente estudo, avaliando a prevalência de AV diminuída em escolares, concluiu que há falhas neste diagnóstico e na continuidade da assistência oftalmológica, tornando-se imprescindível a implantação de um programa público de saúde ocular para reduzir suas consequiências negativas ${ }^{(13)}$. Neste sentido, as campanhas promovidas por serviços de residência médica colaboram para a prevenção e detecção de muitas doenças oculares e aliviam momentaneamente a demanda reprimida de soluções médicas, mas não diminuem a necessidade de programas governamentais de maior amplitude e com mais recursos para este objetivo. 


\section{ConclusÃo}

As campanhas oftalmológicas têm o intuito principal de beneficiar um grande número de pessoas carentes. Para isso, as mesmas devem ser formuladas adequadamente. Fatores como população alvo, local de atendimento, disponibilidade de equipamentos, oferta de transporte, triagem prévia e possibilidade de encaminhamento de pacientes para serviço especializado devem ser considerados.

Campanhas realizadas no próprio local da triagem mostraram maiores índices de comparecimento, mas menor capacidade de resolutividade dos problemas encontrados. A alta taxa de absenteísmo ao atendimento, quando em local distante dos pontos de triagem, provavelmente deveu-se à falta de transporte ao hospital ou falta de estímulo ao comparecimento.

As medidas da AV aferida por equipe de saúde previamente treinada pelo curso mostraram baixo índice de falso-positivo para redução da AV, indicando triagem prévia adequada.

Os atendimentos foram altamente resolutivos no sentido de que a maioria dos casos teve seus óculos prescritos e doados aos necessitados e pequena parcela dos pacientes atendidos nas três campanhas necessitaram consultas eletivas por apresentarem achados que requeriam assistência além dos propósitos daqueles momentos.

\section{Abstract}

Purpose: Ocular health promotion activities are increasingly performed by medical institutions in order to provide assistance for patients in need and to promote blindness prevention. To meet these aims, it is necessary to organize the activity considering all the staff and equipment involved, and the logistic in the process. The present study analyses three ophthalmological programs to identify the factors related to a successful social program. Methods: Observational descriptive study including three ocular health campaigns carried out in 2005. Results: The following campaigns were included: (1) Ophthalmological care for students of a public school and their relatives. Low visual acuity was detected in screening in the school in 90 patients, but only $45.5 \%$ of those showed at the hospital. Optical glasses were prescribed in $73 \%$ of the patients and $14.5 \%$ needed to be referred to ambulatory care; (2) Glaucoma screening in an agribusiness event. Of a total 107 examined individuals, none case of suspicious glaucoma was detected, but large information about how to detect precociously the disease, was done; (3) Visual acuity measurement and refraction of 1.200 school children, without previous screening. Were prescribed and donated ocular glasses for $18.83 \%$ of the examined children. Conclusion: Factors like the place for ophthalmological examination (community or hospital), free transportation, previous screening, available equipment and center for patients' referrals are related to higher efficiency and resolutivity of ocular health programs.

Keywords: Health promotion; Triage; Ocular health; Visual acuity; School health

\section{REFERÊNCIAS}

1. Lopes GJA, Casella AMB, Chuí CA. Prevalência de acuidade visual reduzida nos alunos da primeira série do ensino fundamental das redes pública estadual e privada de Londrina-PR, no ano de 2000. Arq Bras Oftalmol. 2002; 65(6):659-64.

2. Lauretti Filho A, Romão E. Estudo da acuidade visual e dos vícios de refração em crianças com baixo rendimento escolar. Rev Bras Oftalmol. 1982; 41(5):31-6.

3. Zamberlam FRRS. Saúde ocular de escolares de quinta a oitava série do ensino fundamental de uma escola da periferia de Avaré - SP - Brasil. Rev Bras Oftalmol. 2002; 61(1):50-3.

4. Kara-José N, Temporini ER. Avaliação dos critérios de triagem visual de escolares de primeira série do primeiro grau. Rev Saúde Pública. 1980; 14(2):205-14.

5. Temporini ER. Ação preventiva em problemas visuais de escolares. Rev Saúde Pública. 1984;18(3):259-62.

6. Ariyasu RG, Lee PP, Linton KP, LaBree LD, Azen SP, Siu AL. Sensitivity, specificity, and predictive values of screening tests for eye conditions in a clinic-based population. Ophthalmology. 1996;103(11):1751-60. Comment in: Ophthalmology. 1997;104(9):1369-70.

7. Temporini ER, Kara-José N, Taiar A, Ferrarini ML. Validade da aferição da acuidade visual realizada pelo professor em escolares de $1^{\mathrm{a}}$ à $4^{\mathrm{a}}$ série de primeiro grau de uma escola pública do município de São Paulo, Brasil. Rev Saúde Pública. 1977;11(2):229-37.

8. Schimiti RB, Costa VP, Gregui MJF, Kara-José N, Temporini ER. Prevalence of refractive errors and ocular disorders in preschool and schoolchildren of Ibiraporã-PR, Brazil (1989 to 1996). Arq Bras Oftalmol. 2001; 64(5):379-84.

9. Quigley HA, Park CK, Tracey PA, Pollack IP. Community screening for eye disease by laypersons: the Hoffberger program. Am J Ophthalmol. 2002;133(3):386-92.

10. Schellini SA, Shimoda GA, Hisano C, Utyama LAC, Silva MRBM. Estudo da prevalência das afecções oculares nos pré-escolares da cidade de Botucatu (São Paulo). Rev Bras Oftalmol. 1987;46(2):21-6.

11. Póvoa CA, Nicolela MT, Valle ALSL, Gomes LES, Neustein I. Prevalência de glaucoma identificada em campanha de detecção em São Paulo. Arq Bras Oftalmol. 2001;64(4):303-7.

12. Ghanem CC. Levantamento de casos de glaucoma em Joinville - Santa Catarina, 1984. Arq Bras Oftalmol. 1989;52(2):40-3.

13. Gianini RJ, Masi E, Coelho EC, Oréfice FR, Moraes RA. Prevalência de baixa acuidade visual em escolares da rede pública, Sorocaba. Rev Saúde Pública. 2004;38(2):201-8.

\section{ENDEReÇo PARA CoRRESPONDÊNCIA: \\ Dr. João Borges Fortes Filho \\ Curso de Especialização em Oftalmologia \\ Hospital Banco de Olhos de Porto Alegre \\ Rua Eng. Walter Boehl, 285 - Bairro Vila Ipiranga \\ CEP 91360-090 - Porto Alegre - RS}

Tel: + 55 (51) 3344-1972/ Cel + 55 (51) 9969-8081

Fax: + 55 (51) 3222-9292

www.cursohbo.com.br

E-mail: jbfortes@cursohbo.com.br 